\title{
Quantized Turbulence Physics
}

\author{
Demosthenes Kivotides and Anthony Leonard \\ Graduate Aeronautical Laboratories, California Institute of Technology, Pasadena, California 91125, USA
}

(Received 22 January 2003; published 13 June 2003)

\begin{abstract}
We elaborate the physics of systems of unconstrained, reconnecting vortex filaments with dynamic finite cores of uniform ("quantized") circulation interacting via Biot-Savart and viscous forces. The phenomenology of this purely structured turbulent system includes an inertial range with Kolmogorov's $k^{-5 / 3}$ scaling for the energy spectrum, as well as Kolmogorov's linear in $r$ scaling for the third order longitudinal structure function.
\end{abstract}

DOI: 10.1103/PhysRevLett.90.234503

PACS numbers: 47.27.Ak, 47.27.Gs, 47.32.Cc

The contribution of coherent structures to the statistics of turbulent flow comprises a central problem in turbulence physics. Adopting a vorticity representation of fluid flow, one can define coherent structures to be vorticity patterns (e.g., Burgers's vortices) characterized by a number of parameters (e.g., the core radius to length ratio in case of a filament). In order for such patterns to persist in time their interactions should only cause their transition from one characteristic parameter range to another without simultaneous change of their mathematical definition. Examples of such structures are described in [1]. In this Letter we develop a novel turbulence model in order to address the following question: is there a kind of low dimensional coherent structure capable of representing the dynamically important vorticity field as a collection of its manifestations?

Our approach to the question above is inspired from previous efforts to quantize classical turbulence [2] and is motivated by drawing an analogy with quantum fluids [3]. In particular, starting from the Gross-Pitaevskii model of superfluids and using the Madelung transformation the superfluid dynamics reduce to the inviscid Euler equation, with the additional constraint that the vorticity in the flow must be exclusively in the form of vortex filaments with quantized circulation [4]. This extra constraint is a pure quantum mechanical effect. Although in classical fluids such quantization constraints are absent, the following questions are legitimate: How useful would it be (in turbulence theory) to imagine a Navier-Stokes fluid with its circulation quantized in the same manner as in superfluids? Can one construct a Navier-Stokes analog of the Euler superfluid vortices? In this Letter we respond to the questions above by formulating a heuristic quantization of the Navier-Stokes equation. We propose a turbulence model that depicts unconstrained, reconnecting vortex filaments with dynamic finite cores of uniform (quantized) circulation, interacting via inertial and viscous forces. This formulation is much more complicated than previously suggested vortex models of inertial range and fine scale turbulence [5-9]. These had to assume a specific vortex structure of unknown stability analysis and had to ignore the strong interactions between the vortices, as well as their reconnections. These simplifications are in oblique contrast to the complexity of turbulence, and there is little hope to expect that statistical mechanics obtained from such models could unlock essential turbulence physics. Thus, this Letter extends the previous studies by avoiding many of their simplifications.

If $\boldsymbol{r}_{i}$ is the three dimensional representation of the centerline curve of filament $i$ then the vortex motion is described by

$$
\frac{d \boldsymbol{r}_{i}}{d t}=\boldsymbol{V}\left(\boldsymbol{r}_{i}(t), t\right)
$$

where $\boldsymbol{V}\left(\boldsymbol{r}_{i}(t), t\right)$ is the Biot-Savart velocity:

$$
\boldsymbol{V}(\boldsymbol{x}, t)=-\frac{1}{4 \pi} \int \frac{\left(\boldsymbol{x}-\boldsymbol{x}^{\prime}\right) \times \boldsymbol{\omega}\left(\boldsymbol{x}^{\prime}\right) d \boldsymbol{x}^{\prime}}{\left|\boldsymbol{x}-\boldsymbol{x}^{\prime}\right|^{3}}
$$

with $\boldsymbol{\omega}\left(\boldsymbol{x}^{\prime}\right)$ the vorticity vector. In the quantum Euler case the vorticity $\boldsymbol{\omega}\left(\boldsymbol{x}^{\prime}\right)$ is a delta function along the curve of the filament $C_{i}$ since the superfluid vortices have (at hydrodynamic scales) infinitesimal core sizes. However, in the classical Navier-Stokes case the vortices have dynamic, finite cores, and the vorticity is distributed. This results in a more complex vorticity representation [10]:

$$
\begin{aligned}
\boldsymbol{\omega}\left(\boldsymbol{x}^{\prime}, t\right)= & \sum_{i} \Gamma \int_{C_{i}} \frac{1}{\sigma_{i}(s, t)^{3}} \zeta\left(\frac{\left|\boldsymbol{x}^{\prime}-\boldsymbol{r}_{i}(s, t)\right|}{\sigma_{i}(s, t)}\right) \\
& \times\left(\frac{\partial \boldsymbol{r}_{i}}{\partial s}+\frac{\boldsymbol{x}^{\prime}-\boldsymbol{r}_{i}(s, t)}{\sigma_{i}(s, t)} \frac{\partial \sigma_{i}}{\partial s}\right) d s,
\end{aligned}
$$

where $\sigma_{i}(s)$ is the local core radius of filament $i$ and the smoothing kernel $\zeta$ describes the way vorticity spreads around the core centerline. The calculations are done with the high order algebraic kernel of [11]. $\Gamma$ is the circulation strength attributed to all filaments and is the model's analog of the quantum of circulation. The formula shows that the vorticity field has two constituents. The first term of the sum inside the integral sign models the vorticity component along the direction of the filament tangent $\frac{\partial r_{i}}{\partial s}$. This is the only component present in quantum vortices (without the smoothing effect of $\zeta$ ). The second term 
models the vorticity component along the direction $\boldsymbol{x}$ $\boldsymbol{r}_{i}(s)$ and is induced by the change of $\sigma_{i}(s)$ along the filaments.

In the numerical calculations each filament is divided into a number of finite segments using a set of discretization points $s^{j}$, where $j=1, N$ and $s$ is the arclength parametrization. The time integration is done with a low storage, third order acccurate Runge-Kutta method [12]. Both filament stretching due to the Biot-Savart velocity field and viscous action change $\sigma_{i}$. The former effect has been taken into account by imposing for every discretization point $s^{j}$ the conservation of vortex tube volume:

$$
\frac{d}{d t}\left(\pi \sigma_{i}^{2}\left(s^{j}\right)\left|x\left(s^{j}\right)-x\left(s^{j+1}\right)\right|\right)=0,
$$

where $s^{j+1}$ is the immediate neighbor of point $s^{j}$. The viscous effect is handled by the core-spreading method [13]:

$$
\frac{d \sigma_{i}^{2}}{d t}=2 \gamma \nu
$$

where $\nu$ is the kinematic viscocity and $\gamma$ is a factor depending on the particular kernel $\zeta$ employed and equal to $\gamma_{a}=2.205$ for our choice of kernel. When two filaments approach closer than a fraction of their corresponding core radii, they reconnect. The details of the algorithm can be found in [14] where it was shown to predict adequately the changes in topology, the excitation and propagation of Kelvin waves, the viscous decay of kinetic energy, and the helicity dynamics. An important consequence of circulation quantization is a great simplification in reconnection physics. Reconnections between vortex rings of variable circulation are much more difficult to model efficiently. Periodicity was enforced with the minimum image method [15]. In the absence of a proper mathematical analysis of the error committed with the latter method we have done computational experiments with a simple system which did not show significant velocity deviations from exact reference velocity profiles.

The initial condition of the calculations done with the proposed model consists of 192 vortex rings in a periodic box. The radii and orientations of the rings are chosen using sequences of random numbers. The Reynolds number has the value $\operatorname{Re}=\frac{\Gamma}{\nu}=5000$ where $\Gamma$ is the circulation of the rings and $\nu$ is the kinematic viscosity. The results are made dimensionless in the following manner: $t=\frac{\Gamma t^{\prime}}{R^{2}}, x=\frac{x^{\prime}}{R}, \omega=\frac{R^{2} \omega^{\prime}}{\Gamma}$, where $t^{\prime}, x^{\prime}, \omega^{\prime}$ are dimensional and $R$ is a reference initial vortex-ring radius. We have chosen $\Gamma=1$ and $R=1$; the box size is $l_{b}=2.041$. Using the initial value of the turbulence intensity $u=\sqrt{2 E / 3}=$ 3.87 (with $u_{i}$ the velocity fluctuations and $E=$ $\frac{1}{2} \sum_{i=1}^{3}\left\langle u_{i} u_{i}\right\rangle$ the turbulence kinetic energy) as a scale for the velocity of convective motions and the size of the largest resolvable eddies (equal to half the box size) as a scale for the length of the convective motions, we find the inertial time scale $t_{c}=0.26$. For comparison the numerical solution ends at $t_{e}=0.14$.

Figure 1 shows that the artificially ordered initial condition evolves (mainly because of reconnections) into a complex tangle. The final reconnection number exceeds 9000 .

Figure 2 presents the energy spectra at two different times $t=0.09$ and $t_{e}=0.14$. They lead to the same conclusions, although due to turbulence decay the earlier spectrum is characterized by higher energy values. Notice that the initial condition corresponds to a nonphysical tangle state and only at $t \approx 0.05$ (when an almost linear decay sets in) is the tangle complex enough to be realistic. At $t_{e}=0.14$ the minimum tube radius in the system is located at $k_{h}=14$ and the maximum (tube radius) at $k_{l}=8.25$. Here $k=\frac{1}{l}$ without $2 \pi$ factors. We estimated the average dissipation $\langle\epsilon\rangle=\frac{d E}{d t}$ by equating it to the almost constant turbulent energy decay rate (Fig. 3)
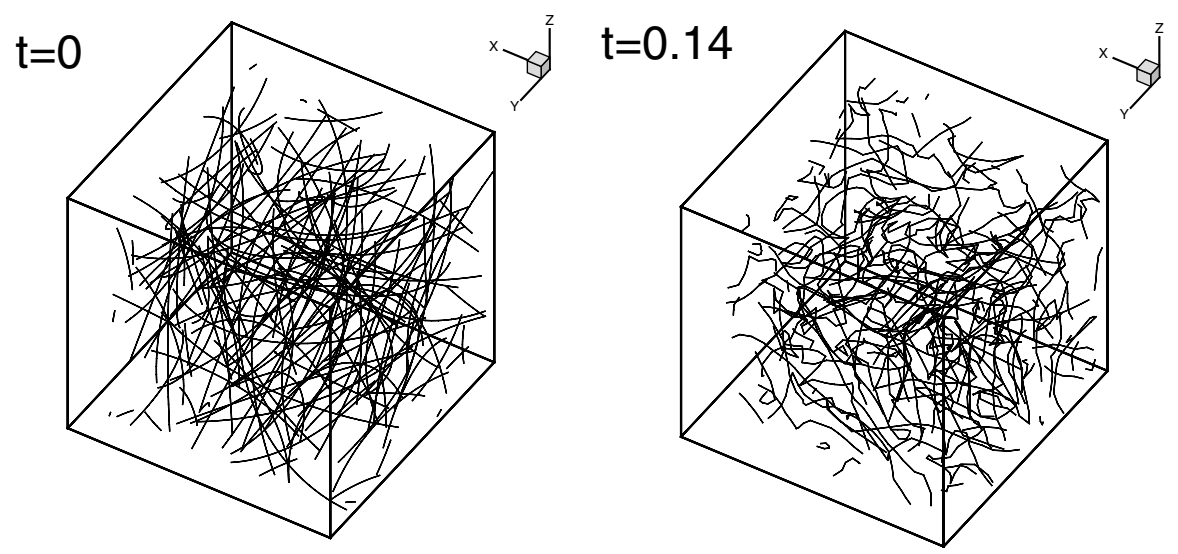

FIG. 1. Vortex filament core centerlines at initial and stoppage times. For clarity, only one-eighth of the computational box is shown. 


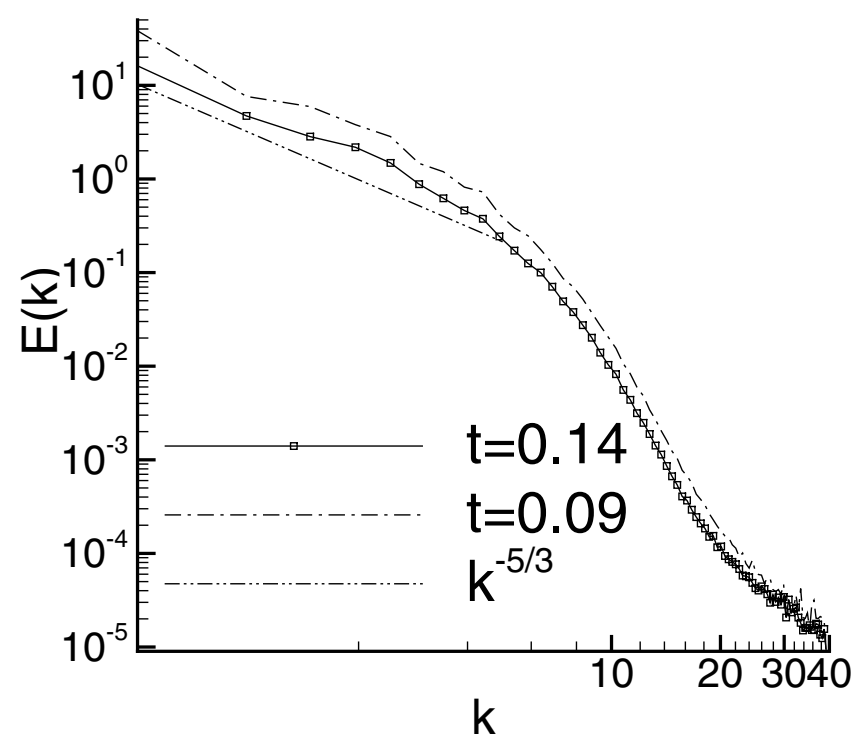

FIG. 2. Velocity spectra at $t=0.09$ and $t_{e}=0.14$.

in the time interval $t=0.07$ to $t=0.14$ and found $\langle\epsilon\rangle=$ 42. Direct calculation using the $\epsilon$ definition is not appropriate since the major contribution to $\epsilon$ comes from the reconnection model and not from the resolved flow scales. Assuming the formula $\langle\epsilon\rangle=\frac{15 \nu u^{2}}{\lambda^{2}}$ (with $\lambda$ the Taylor scale) of locally isotropic turbulence to be useful in the present context, we find the Taylor wave number $k_{\lambda}=37$ and the Taylor scale Reynolds number $\operatorname{Re}_{\lambda}=427$. The Kolmogorov microscale is much smaller than the minimum core size and is not resolved. The $k<k_{l}$ part of the spectrum corresponds to the inertial range of turbulence where vortex stretching is dominant. At $k \approx k_{l}$ viscous effects become important and cause a sharp cutoff observed between $k \approx k_{l}$ and $k \approx k_{h}$. There is also evidence

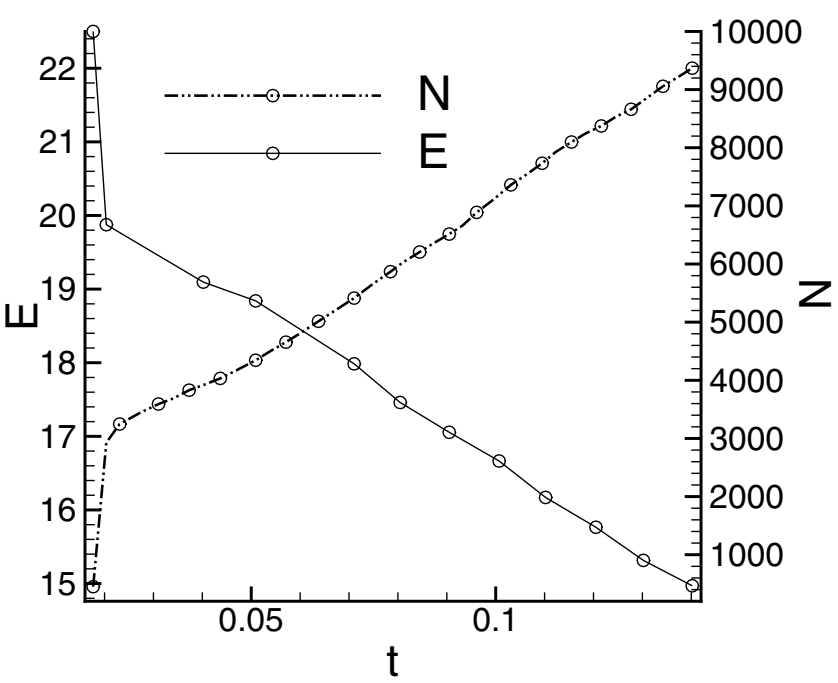

FIG. 3. Evolution of turbulent energy $E$ and of reconnection number $N$. that at $k \approx k_{\lambda}$ energy tends to pile up at subcore wave numbers.

The fully resolved calculations of $[16,17]$ depicted vortex stretching at subcore length scales during reconnections. It was shown in [14] that the intensity of this stretching process is only partially captured by the employed reconnection model, and therefore the small scale flow structure is not predicted with great accuracy. However, assuming that the physics of the inertial range depend mainly on the effective removal of kinetic energy at the smallest wave numbers and not on their detailed structure, this should not present a problem since our model incorporates such an effective dissipation. As Fig. 2 indicates, the inertial range spectrum scales like $k^{-5 / 3}$ and this provides clear evidence that stretching effects in turbulent vortex tangles can help explain Kolmogorov scalings in accord with a line of thought initiated in [18].

In Fig. 3 we observe a direct correlation between the reconnection rate and the (turbulent) energy decay rate. This is also consistent with the findings of [16], showing intensification of dissipation during reconnections. The results hint at a possible mechanism of dissipation intermittency in real turbulence since reconnection processes occupy at any instant only a portion of the fluid volume.

In Fig. 4 the third order longitudinal structure function is shown. The latter is defined as

$$
S_{3}^{\|}=\left\langle\left\{[\boldsymbol{u}(\boldsymbol{x}+\boldsymbol{r})-\boldsymbol{u}(\boldsymbol{x})] \cdot \frac{\boldsymbol{r}}{r}\right\}^{3}\right\rangle,
$$

and it is negative. We have first computed the $S_{3}^{\|}$values along each of the three Cartesian directions and then we averaged. The directional sets of data were not identical

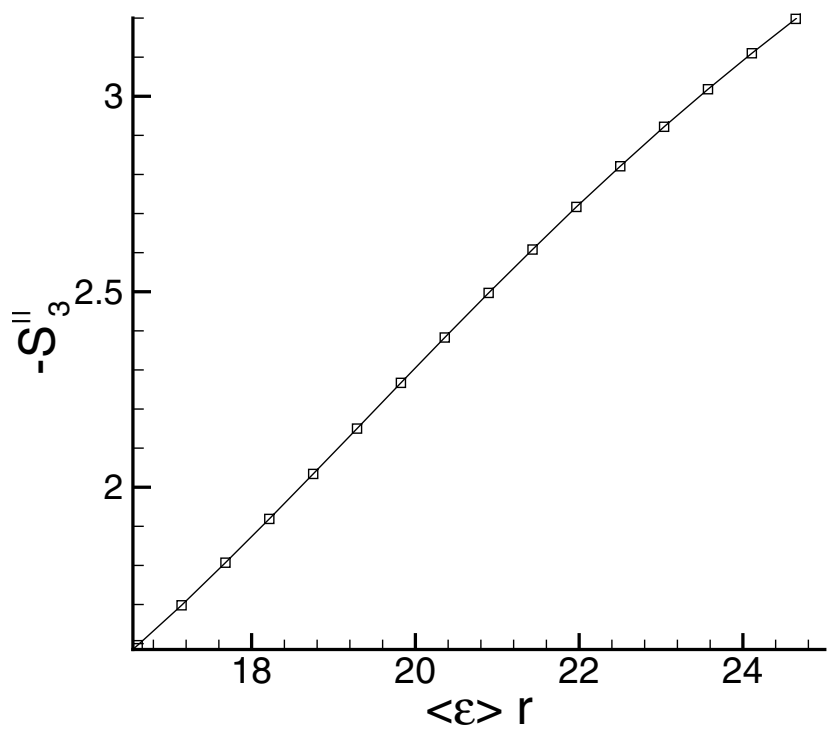

FIG. 4. Third order velocity structure function at stoppage time $t_{e}=0.14$; the actual $r$ interval is $[0.4,0.6]$. 
and the present turbulence is certainly not isotropic. The results indicate that (as in real turbulent flows) $S_{3}^{\|}$is nonvanishing. In addition, $S_{3}^{\|} \propto r$ in a subinterval of the $k^{-5 / 3}$ range. This scaling behavior was apparent in all directions although its extent was varying. The slope, however, in Fig. 4 differs significantly from the Kolmogorov slope $-\frac{4}{5}\langle\epsilon\rangle$ valid for globally isotropic turbulence and calculated using the $\langle\epsilon\rangle=42$ value. Possible reasons for this could be turbulence unsteadiness and anisotropy, a small extent of the inertial range which is not asymptotically distant from the viscous regime, as well as limitations of the present model of fully developed Navier-Stokes turbulence. In this context it is worth noticing that in the grid generated turbulence experiment of [19] with the $\operatorname{Re}_{\lambda} \approx 450$ similar to ours, there was also almost no range with the slope $-\frac{4}{5}\langle\epsilon\rangle$ at all.

In conclusion, we have established a schoinoidal (from the Greek $\sigma \chi о \iota \nu o \epsilon \iota \delta \eta \varsigma$ meaning ropelike, stringy) kind of turbulence. In doing this we have ignored the incoherent background vorticity of real turbulence and consequently the interaction between the latter and the vortex filaments. In this milieu, it is important that the system exhibits the Kolmogorov $k^{-5 / 3}$ scaling, as well as the Kolomogorov $S_{3}^{\|} \propto r$ prediction, despite the turbulence being highly intermittent and anisotropic. These do not necessarily mean that stretched line vortices are the sole factor of Kolmogorov phenomenology. The latter preassumes (among other equally plausible alternatives) that real turbulence is solely composed of linear coherent structures (as is the case of quantum turbulence) or that filamentary structures dominate an apparently incoherent background. Arguments against the latter can be found in [20]. The existence of different turbulence species all with the same statistical phenomenology but different deterministic structures should not be excluded. In this milieu, the study of hybrid systems of filament, sheet, and volume vorticity is important. In the related area of quantum fluids the results help to understand the $k^{-1}$ [3] scaling of superfluid turbulence by noting that vortex stretching, a necessary (in this case) factor of
Kolmogorov scaling is missing in the coreless quantum vortex tangles.

This research was partially supported by the Office of Naval Research and the Department of Energy.

[1] K. Horiuti, Phys. Fluids 13, 3756 (2001).

[2] K. Kondo, RAAG Memoirs 2, 71 (1958).

[3] D. Kivotides, J.C. Vassilicos, D. C. Samuels, and C. F. Barenghi, Europhys. Lett. 57, 845 (2002).

[4] Quantized Vortex Dynamics and Superfluid Turbulence, edited by C. F. Barenghi, R. J. Donnelly, and W. F. Vinen (Springer-Verlag, Berlin, 2001).

[5] D. I. Pullin and P. Saffman, Annu. Rev. Fluid Mech. 30, 31 (1998).

[6] N. Hatakeyama and T. Kambe, Phys. Rev. Lett. 79, 1257 (1997).

[7] D. Boyer and J.C. Elicer-Cortes, J. Phys. A 33, 6859 (2000).

[8] D. I. Pullin and T. S. Lundgren, Phys. Fluids 13, 2553 (2001).

[9] A. J. Chorin, Vorticity and Turbulence (Springer-Verlag, Berlin, 1994).

[10] A. Leonard, Phys. Fluids 6, 765 (1994).

[11] G. S. Winckelmans and A. Leonard, J. Comput. Phys. 109, 247 (1993).

[12] P. Orlandi, Fluid Flow Phenomena-A Numerical Toolkit (Kluwer Academic Publishers, Amsterdam, 1999).

[13] A. Leonard, Annu. Rev. Fluid Mech. 17, 523 (1985).

[14] D. Kivotides and A. Leonard (to be published).

[15] P. Susanne and P. Gibbon, Many-Body Tree Methods in Physics (Cambridge University Press, Cambridge, U.K., 1996).

[16] P. Chatelain, D. Kivotides, and A. Leonard, Phys. Rev. Lett. 90, 054501 (2003).

[17] M. J. Shelley, D. I. Meiron, and S. A. Orszag, J. Fluid Mech. 246, 613 (1993).

[18] G. I. Taylor, Proc. R. Soc. London A 164, 15 (1938).

[19] L. Mydlarski and Z. Warhaft, J. Fluid Mech. 320, 331 (1996).

[20] A. Tsinober, An Informal Introduction to Turbulence (Kluwer Academic Publishers, Amsterdam, 2001). 